\title{
Knowledge Extraction from Neural Networks
}

\author{
Antony Browne, Brian Hudson, David Whitley, Martyn Ford, Phil Picton and Hassan Kazemian
}

\begin{abstract}
In the past, neural networks have been viewed as classification and regression systems whose internal representations were incomprehensible. It is now becoming apparent that algorithms can be designed which extract comprehensible representations from trained neural networks, enabling them to be used for data mining, i.e. the discovery and explanation of previously unknown relationships present in data. This paper reviews existing algorithms for extracting comprehensible representations from neural networks and describes research to generalize and extend the capabilities of one of these algorithms. The algorithm has been generalized for application to bioinformatics datasets, including the prediction of splice site junctions in Human DNA sequences. Results generated on this dataset are compared with those generated by a conventional data mining technique (C5), and conclusions are drawn regarding the application of the neural network based technique to other fields of interest.
\end{abstract}

Index Terms-Neural networks, knowledge extraction, data mining, bioinformatics.

\section{I. INTRODUCTION}

Data mining (or more precisely, knowledge extraction) can be described as the process of discovering previously unknown dependencies and relationships in data sets. As Craven and Shavlik [12] observe: "A (learning) system may discover salient features in the input data whose importance was not previously recognized." In the past, most data mining has been performed using symbolic artificial intelligence data algorithms such as $\mathrm{C} 4.5$ and $\mathrm{C} 5[21,44]$ or CART [6]. Neural Networks (NNs) have traditionally been treated as 'black boxes', because most NNs do not use easily interpretable representations such as rules or decision trees. A fundamental

Manuscript received July 15 th 2003 . This work was supported in part by the UK Biotechnology Biological Sciences Research Council and the Engineering Physical Sciences Research Council (grant ref: 322/BIO12005) and SPSS UK Ltd.

A. Browne is with the Computing Department, University of Surrey, Guildford GU2 7XH, UK (e-mail: a.browne@ surrey.ac.uk)

B. Hudson, D. Whitley and M. Ford are with the Centre for Molecular Design, University of Portsmouth, UK

P. Picton is with the School of Technology and Design, University College Northampton, UK.

H. Kazemian is with the Department of Computing, Communications Systems and Mathematics, London Metropolitan University, London, UK question is: "Why bother extracting decision trees from NNs, when symbolic data mining techniques exist?" It is obviously worthwhile data mining with NNs if some increase in performance is observed, such as:

- The decision tree/rule set extracted by the $\mathrm{NN}$ technique gives a better fit to test data than symbolic techniques, indicating that it has more closely modeled the task being studied.

- The decision tree/rule set extracted by the NN technique is represented in a more comprehensible way to the client group viewing the results of the data mining. For some domains, particular ways of representing the extracted knowledge may be more comprehensible than others.

Obviously, a trained $\mathrm{NN}$ has learned interesting relationships inherent in the data set it was trained on. However, these relationships are encoded as weight vectors within the trained $\mathrm{NN}$, which are difficult to interpret (particularly because of non-linearities present in the network transfer functions). Statistical techniques exist to analyze the weight vectors representing trained networks (for a review of statistical techniques see Bullinaria [9] or for alternative methods see [7]) but such techniques can be difficult for nonstatisticians to interpret. Structures such as rules and decision trees are a more comprehensible way of presenting information to other audiences. In recent years, researchers have developed techniques to extract easily interpretable structures, such as symbolic IF-THEN rules and decision trees, from NNs once they have been trained (for a review of these techniques, see $[1,2,8,32])$. Some of these approaches require specialized restricted weight modification algorithms [28], whilst others require specialized network architectures such as an extra hidden layer of units with staircase activation functions [4]. Other approaches extract rules by observing the relationship between the NN's inputs and outputs. Because of this, the latter algorithms are general purpose in nature and can be applied to any feed forward network architecture [12]. An alternative to the IF-THEN form of rules exists, the M-of-N rule, which (this paper argues) may be more suitable for some applications as it confers superior understanding of the problem domain to the relevant audiences (see justification later). Rules in this form state: 'If $\mathrm{M}$ of the $\mathrm{N}$ conditions $\mathrm{a}_{1}, \mathrm{a}_{2}, \ldots, \mathrm{a}_{\mathrm{m}}$, are true, then the 
conclusion $b$ is true'. It is argued that some concepts can be better expressed in such a form, and use of this form also help avoid the combinatorial explosion in tree size found with IFTHEN rules. A flexible data mining algorithm operating on NNs and using the M-of-N rule representation is the TREPAN algorithm $[11,12]$ which is so flexible that it (theoretically) is not restricted to feed-forward NNs, it could also be applied to other systems such as standard statistical classifiers. Initial results on biological datasets [11] suggested that TREPAN has potential in bioinformatics domains. For example, in a protein coding problem domain, TREPAN produced better performance on a test set, giving 61 percent accuracy to a test set versus 55 percent for $\mathrm{C} 4.5$, and produced more comprehensible decision trees, with five internal nodes and 14 feature references (the number of input variable from the data set incorporated into the decision tree) versus 53 internal nodes and 53 features for C4.5.

\section{IMPLEMENTATION OF A GENERALIZED TREPAN ALGORITHM}

The original implementation was restricted to extracting decision trees from a limited class of MLPs (a simple MLP trained by the conjugate gradients algorithm, with no regularization built into the algorithm), precluding the use of more general NNs or other classification methods. The algorithm was therefore completely recoded in the MATLAB [19] programming environment. During the recoding, attention was paid to generalization of the code, in particular a major requirement was that the software should be compatible with any relevant classifier (i.e. it could be 'bolted on' to any feedforward classification methodology). The only requirement was that a MATLAB function could be provided for the classifier that the algorithm was to be linked with, such that the function would take a pattern to be classified as input and produce a classification as output. In its current form the tool links seamlessly to NNs developed using the public-domain NETLAB [20] toolbox, but can easily be adapted to those developed in the proprietary MATLAB NNs toolkit (and indeed it takes little effort for networks coded in languages other than MATLAB to be used). Such an architecture will allow the tool to be used with other 'black box' models, such as Hidden Markov Models (HMMs) [22] and Support Vector Machines (SVMs) [34]. The tool builds an M-of-N based decision tree representing the function that the NN has learnt, by recursively partitioning the input space. The algorithm draws query instances by taking into account the actual distribution of training data instances in the problem domain. This model of the training data is used in a generative manner to draw instances of input vectors for presentation to the network. The decision tree is built in a best-first manner, in that as each node is added to the decision tree the algorithm tries to maximize the gain in fidelity of the extracted decision tree to the network it is trying to model. Stopping criteria can be selected so that the size of the tree returned can be controlled by the user, aiding comprehensibility. The decision tree extracted is stored as an array of node structures. The topology of the tree is determined by assigning an index to each node, with the root node having index 1 , and each node storing the indices of its parent and children. Binary splitting tests are used at the nodes, so that each node has either zero or two children. A node with no children is a leaf; a node with two children is a fork. The class assigned by the tree to examples reaching a node is determined by majority vote: the class with the largest number of training examples reaching the node wins. This tool differs from other decision tree algorithms in the three significant areas discussed below.

Firstly, the decision tree is calculated using a best-first method for selecting the order in which nodes of the tree are expanded. Each node is assigned a priority, defined to be the proportion of examples misclassified by the node. The algorithm maintains a queue of leaf nodes, ordered by their priority, and successively expands the node at the head of the queue into a fork with two children. Nodes with higher priorities are processed first, as they offer the greatest chance of increasing the number of correctly classified training examples. New child nodes are placed in the queue if they have non-zero priority; i.e. some of the examples reaching the node are classified incorrectly. Both the tree and the queue are initialized with the root node, which all the training examples pass through. The process stops when the number of nodes in the tree exceeds some pre-defined maximum size, or when the queue is empty.

A second distinguishing feature is that the splitting tests used at the nodes are $\mathrm{M}$-of- $\mathrm{N}$ tests, defined by two integers $\mathrm{M}$ and $\mathrm{N}$, and a set $\mathrm{S}$ of $\mathrm{N}$ splits, where a split is a Boolean test on an individual feature. An $\mathrm{M}$-of-N test (M, N, S) is satisfied if at least $\mathrm{M}$ of its $\mathrm{N}$ splits are true. During classification, when an example reaches a fork in the tree it passes down the first, left-hand branch if the M-of-N test at that node is satisfied, and down the second, right-hand branch otherwise. The choice of which $\mathrm{M}$-of- $\mathrm{N}$ test to use at a node is based on maximizing the information gain [26] produced by the test. To avoid the potential combinatorial explosion involved in searching the space of all possible M-of- $N$ tests, a beam search [18] is used. The beam is initialized with the split having the largest information gain and its complement. The beam search proceeds iteratively, constructing increasingly complex M-of$\mathrm{N}$ tests for possible inclusion in the beam, terminating when the beam remains unchanged during a single iteration. To avoid over-fitting the training data, these tests are considered for inclusion in the beam only if the partitioning of the training set they produce is significantly different (on the basis of a $\chi^{2}$ test) from that produced by the original M-of- $N$ test. A test that satisfies this condition replaces a test in the current beam if it has a larger information gain. A common limitation of decision tree methods is that as the tree grows, the number of training cases reaching a node decreases, until there are inadequate examples to expand the tree further.

The third, and perhaps most notable aspect of the tool aims to overcome this: the algorithm generates additional input 
patterns by sampling the empirical distributions of the original training data. These additional generated training cases are used alongside the original training data to expand the tree. As each node is created, sufficient training cases are generated so that a pre-specified mini-mum number of examples reach the node. The generation of new input patterns is based only on the marginal distributions of the features, and ignores any correlations between the features. To partially address this deficiency the tool estimates the local distributions of the features at each node (i.e. the empirical distributions of the original training cases that reach the node). If these distributions are significantly different (again on the basis of a $\chi^{2}$ test) from the distributions at the parent node, then the local distributions are used to generate new input patterns; otherwise the parent distributions are used.

\section{RESULTS}

The algorithm has been applied an example from bioinformatics, chosen based on a number of criteria, with perhaps the most important of these being that the example was well known and the solution previously identified. This example involves the prediction of splice junction sites in human DNA sequences, where sequences are composed of base-pairs (bp) of four bases, signified by the letters A, C, G and $T$. The subject is important because the ability to identify the location of these sites is crucial for the performance of the gene finding programs. These programs attempt to predict whether a particular DNA sequence contains a gene coding for a protein and, if so, to predict the likely gene product. The problem has been extensively studied using a variety of different methodologies [23, 40, 43] and the results have been compared [10]. Splice junctions are the positions at which, after primary transcription of the DNA into RNA, the noncoding regions (introns) of a gene are excised to form edited m-RNA. This is eventually used to provide the template for protein translation. Donor (Exon/Intron) sites are nearly always located immediately preceding a GT sequence, thus GT pairs within a DNA sequence are markers for potential splice junction sites. The task is therefore to identify which of these potential sites correspond to real sites, and hence to make predictions of likely genes and gene products. The dataset used here was that prepared by Thanaraj $[26,27]$. This was a 'clean' dataset of 641 sequences from Human genes, each of which has been confirmed by experiment using expressed sequence tags. In the dataset there are 567 donor sites, which have been used as the training set positives in the simulations below. The window used for the donor junctions was a 9 bp sequence (coded as 1 of $n$ binary inputs where $n=4$ ), starting at position -3 and ending at position +6 (where the GT marker is at positions +1 and +2 ). Negative training set examples were generated using recommended procedures [27]. These rely on the observation that GT markers lying close to a real splice junction are much less likely to be another real junction, and so false sites can be generated by taking sequences close to confirmed real sites. Using this method, false sites were generated from both upstream and downstream sides of the real sites, giving 943 false donors. In order to determine the architecture of the MLPs to be used the training set was split into training/validation/test sets in the ratio 50:25:25. Using empirical techniques network architectures using a single hidden layer of ten units were selected for the donor problem. Once selected, the full training set was used to train five networks using the specified architecture. Testing was evaluated using the test set from [30] which is a set of sequences developed using the same rules, and subject to the same criteria, which were identified after the main set had been developed. Using the same procedures as for the training set, donor test sets were produced comprising 229 true and 373 false sequences. The decision trees for each of the networks for the donor problem all converged to the same results and produced the single M-of-N rule shown below, followed by the corresponding decision tree produced by $\mathrm{C} 5$. The tree extracted from or neural network gave 90.7 percent correct generalisation to the test set and the $C 5$ tree gave 91.4 percent correct.

$\mathrm{M}$-of-N rule extracted from neural network: 'If Three

of $((p-2)=A ;(p-1)=G ;(p+3)=A ;(p+4)=$

$A ;(p+5)=G)$ then REAL else FALSE'

Decision tree from $\mathrm{C5}$ :

Rules 1..4:

$(\mathrm{p}+5=\mathrm{T})^{\wedge}((\mathrm{p}+3=\mathrm{C}) \vee(\mathrm{p}+3=\mathrm{G}) \vee(\mathrm{p}+3=\mathrm{T}))$ then FALSE

$(p+5=T)^{\wedge}(p+3=A)^{\wedge}(p+2=G)$ then REAL else FALSE

Rules 5..15:

$(\mathrm{p}+5=\mathrm{A}) \wedge((\mathrm{p}+2=\mathrm{A}) \vee(\mathrm{p}+2=\mathrm{C}) \vee(\mathrm{p}+2=\mathrm{T}))$ then FALSE

$(\mathrm{p}+5=\mathrm{A})^{\wedge}(\mathrm{p}+2=\mathrm{G})^{\wedge}((\mathrm{p}+3=\mathrm{C}) \vee(\mathrm{p}+3=\mathrm{G}) \vee(\mathrm{p}$ $+3=\mathrm{T})$ ) then FALSE

$(\mathrm{p}+5=\mathrm{A})^{\wedge}(\mathrm{p}+2=\mathrm{G})^{\wedge}(\mathrm{p}+3=\mathrm{A})^{\wedge}((\mathrm{p}+4=\mathrm{G}) \vee(\mathrm{p}+$ $4=\mathrm{T})$ ) then FALSE

$(\mathrm{p}+5=\mathrm{A})^{\wedge}(\mathrm{p}+2=\mathrm{G})^{\wedge}(\mathrm{p}+3=\mathrm{A})^{\wedge}(\mathrm{p}+4=\mathrm{A})$ then FALSE

$(\mathrm{p}+5=\mathrm{A})^{\wedge}(\mathrm{p}+4=\mathrm{C})^{\wedge}(\mathrm{p}+1=\mathrm{A})$ then REAL else FALSE

Rules 16..25:

$(p+5=C)^{\wedge}((p+2=A) \vee(p+2=C) \vee(p+2=T))$ then FALSE

$(\mathrm{p}+5=\mathrm{C})^{\wedge}(\mathrm{p}+2=\mathrm{G})^{\wedge}((\mathrm{p}+1=\mathrm{C}) \vee(\mathrm{p}+1=\mathrm{G}) \vee(\mathrm{p}+$ $1=\mathrm{T})$ ) then FALSE

$(\mathrm{p}+5=\mathrm{C})^{\wedge}(\mathrm{p}+1=\mathrm{A})^{\wedge}((\mathrm{p}+3=\mathrm{C}) \vee(\mathrm{p}+3=\mathrm{T}))$ then FALSE

$(p+5=C)^{\wedge}(p+3=A)$ then REAL

$(p+5=C)^{\wedge}(p+3=G) \wedge(p-3=C)$ then REAL else FALSE 
Rules 26..34:

$(p+5=G) \wedge((p+3=C) \vee(p+3=T))$ then FALSE

$(\mathrm{p}+5=\mathrm{G})^{\wedge}(\mathrm{p}+3=\mathrm{A})^{\wedge}(\mathrm{p}+2=\mathrm{G})$ then REAL

$(p+5=G)^{\wedge}(p+2=A)^{\wedge}((p+4=A) \vee(p+4=G))$ then

REAL

$(\mathrm{p}+5=\mathrm{G})^{\wedge}((\mathrm{p}+4=\mathrm{C}) \vee(\mathrm{p}+4=\mathrm{T}))$ then FALSE

$(p+5=G) \wedge(p+2=C)^{\wedge}(p+4=A)$ then REAL else

FALSE

Rules 35..41:

$(\mathrm{p}+2=\mathrm{T})^{\wedge}((\mathrm{p}+6=\mathrm{A}) \vee(\mathrm{p}+6=\mathrm{G}))$ then FALSE

$(p+2=T)^{\wedge}((p+6=C) \vee(p+6=T))$ then REAL

$(\mathrm{p}+2=\mathrm{T})^{\wedge}(\mathrm{p}+3=\mathrm{G})^{\wedge}(\mathrm{p}+4=\mathrm{T})$ then FALSE

$(\mathrm{p}+2=\mathrm{T})^{\wedge}(\mathrm{p}+4=\mathrm{C})^{\wedge}(\mathrm{p}+6=\mathrm{T})$ then REAL else

FALSE

Rules: 42..45:

$(\mathrm{p}+4=\mathrm{A}) \wedge((\mathrm{p}+2=\mathrm{C}) \vee(\mathrm{p}+2=\mathrm{G}) \vee(\mathrm{p}+2=\mathrm{T}))$ then REAL

$(p+4=A)^{\wedge}(p+2=A)^{\wedge}(p-3=T)$ then FALSE else REAL

Rules 46..55:

$(\mathrm{p}+4=\mathrm{G})^{\wedge}((\mathrm{p}+2=\mathrm{A}) \vee(\mathrm{p}+2=\mathrm{C}) \vee(\mathrm{p}+2=\mathrm{T}))$ then FALSE

$(p+4=G)^{\wedge}(p+2=G)^{\wedge}((p+1=A) \vee(p+1=C))$ then REAL

$(p+4=G) \wedge((p+1=G) \vee(p+1=T))$ then FALSE

For comparison, the generally accepted consensus sequence for donor sites [36] is: ' $(\mathrm{C} \vee \mathrm{G}) \mathrm{A} \mathrm{G} \mid \mathrm{G} \mathrm{T}(\mathrm{A} \vee \mathrm{G}) \mathrm{A} \mathrm{G} \mathrm{T}$ '

\section{DISCUSSION}

From the results, it is clear that this dataset fulfills the criterion that the MLP predictions are equal in terms of accuracy of the predictions on a test set to the symbolic rule induction method. Furthermore, the rules extracted from the $\mathrm{NN}$ are simpler than those from $\mathrm{C5}$, with the donor problem being expressed as a single $\mathrm{M}$-of-N rule in contrast to the rather complicated $\mathrm{C5}$ decision tree. The $\mathrm{M}$-of-N rule can be expanded into a maximum number of ten individual rules, whereas the ruleset extracted by $\mathrm{C} 5$ has 55 individual members. Clearly, extracting rules from the neural network aids comprehensibility. It was also noticed that the rules are cast in a form which appears particularly suitable for the representation of these data, where the 'correct' answer is usually expressed in terms of a consensus sequence. This paper argues that this is the main advantage of this tool for biological sequence problems. The consensus sequence is standard practice for molecular biologists wishing to express rules to describe their data, and may be an advantageous way of expressing results for different problem domains. Another potential advantage is that neural nets and HMMs can discover non-linear relationships in the data, and perform significantly better than symbolic rule induction methods in gene finding programs [30]. HMMs have proved highly successful in the bioinformatics arena, and have been incorporated into several gene-finding programs such as GENIE [17], GRAIL [33] and GRAIL EXP [39], that are routinely used by practicing biologists. In situations where these non-linear effects are important, the methodology outlined in this paper shows great promise, and an intriguing possibility is the application of the algorithm outlined in this paper to HMMs. Another very positive conclusion from this work is that the flexibility of the algorithm developed enables its application to other NN based and statistical classifiers, such as SVMs. In addition, as the algorithm only inspects the input-output behaviour of the system, it is ideally suited for application to ensembles of diverse neural net classifiers [27], using techniques such as boosting [13,24], bagging [5] and stacking [38], potentially with the application of different ensemble combination methods [41]. Sampling is an important part of this algorithm. This is the process whereby examples are generated (consistent with the distributions of the real dataset). The objective of the algorithm is to represent the behaviour of the $\mathrm{NN}$, rather than just to predict the results for a test set. The sampling methodology ensures that a sufficient number of examples are presented to the network at each node in the tree. However, it is recognized that this is dependent on having a good distribution of values in the training set to avoid the possibility of bias. This may be especially problematic where the original data is sparse. The algorithm as outlined here at present only accepts nominal inputs. As far as genomic sequence datasets are concerned, this is not a limitation, as all inputs are nominal, but an obvious extension is to build in a sampling mechanism for real-valued features. This is likely to be particularly important in protein sequence problems where, unlike DNA sequences where the alphabet is largely irrelevant to the function, the properties of each residue in the sequence are likely to be much more important than the arbitrary letter assignment. The origina! TREPAN algorithm uses kemel discriminant analysis to sample such features. However, there are problems with using this sampling method [3] and a more sophisticated method such as EM will be used in further implementations of the software. Another factor to consider for datasets consisting of many input features is that in the context of classification, rule extraction from NNs is an NPhard problem [16]. This implies that scaling our algorithm to very large tasks may be problematic (depending on the availability of suitable hardware or access to distributed computing resources). Other techniques exist which scale polynomially, including the Discretized Interpretable Multi Layer Perceptron model [4] and the model of Vaughn [35]. It is possible that these techniques may have to be applied when attempting to extract knowledge from large networks where problem size and hardware availability preclude the application of our algorithm to generate results in an acceptable time frame. However, such techniques are not as general purpose as the tool outlined in this paper. 


\section{CONCLUSIONS}

Modern data gathering techniques are producing vast amounts of data. However, data can be useless in the absence of understanding. The work described here provides a generalized methodology that can address problems in many domains. Preliminary results are promising; in particular there is evidence that the formalism employed may be particularly suitable to the understanding of some problem domains. The combination of NNs with an algorithm to extract knowledge from the trained networks potentially offers the 'best of both worlds' to those attempting to both make predictions on their data and simultaneously understand it. This technique demonstrates that it is possible to combine the generalization accuracy of NNs with the comprehensibility generated by the knowledge extraction method applied to them. As well as facilitating knowiedge discovery, the user obtains understanding of how the network is performing its task. This should lead to both more confidence in and better acceptance of the application of these techniques. The extraction of decision trees from trained NNs is an important addition to the data mining toolkit of knowledge extraction techniques.

\section{REFERENCES}

[1] R. Andrews, A. B. Tickle, M. Golea and J. Diederich, Rule extraction from trained artificial neural networks, in: A. Browne, ed., NN Analysis, Architectures and Algorithms, (Institute of Physics Press, Bristol, UK, 1997).

[2] C. S. Ampratwum, P. D. Picton and A. Browne, Rule extraction from neural network models of chemical species in optical emission spectra, in: Proc. Workshop on Recent Advances in Soft Computing (1998) 5364.

[3] C. M. Bishop, Neural Networks for Pattern Recognition, (Clarendon Press, 1995).

[4] G. Bologna, Symbolic rule extraction from the DIMLP neural network, in: S. Wermter and R. Sun, eds., Neural Hybrid Systems (Springer Verlag, 1999).

[5] L. Breiman, Bagging predictors, Machine learning 26(2) (1996) 123140 .

[6] L. Breiman, J. H. Friedman, R. A. Olshen and C. J. Stone, Classification and Regression Trees (Wadsworth, Belmont, CA, 1984).

[7] A. Browne, Neural Network Analysis, Architectures and Algorithms (Institute of Physics Press, Bristol, UK, 1997).

[8] A. Browne and R. Sun, Connectionist inference models, Neural Networks, vol. 4 , no. 10, pp. 1331-1355, 2001

[9] J. A. Bullinaria, Analysing the internal representations of trained Neural Networks, in: A. Browne, ed., Neural Network Analysis, Architectures and Algorithms (Institute of Physics Press, Bristol, UK, 1997).

[1.0] M. Burset and R. Guigo, Evaluation of Gene Structure Prediction Programs, Genomics, 34 (1996) 353-367.

[11] M. W. Craven, Extracting comprehensible models from trained neural networks, Ph.D. Thesis, University of Wisconsin, Madison (1996)

[12] M. W. Craven and J. W. Shavlik, Understanding time series networks, International Journal of Neural Systems 8(4) (1997) 373-384.

[13] H. Drucker, R. Schapire and P. Simard, Boosting performance in neural networks, International Journal of Pattern Recognition and Artificial Intelligence 7(4) (1993) 705-719.

[14] M. Golea, On the complexity of rule extraction from neural networks and network querying, in: Proc. of the Rule Extraction from Trained Artificial Neural Networks Workshop at the Society for the Study of Artificial Intelligence and the Simulation of Behaviour Workshop Series (AISB, 1996) 51-59.

[15] D. Kulp, D. Haussler, M. G. Reese and F. H. Eeckman, A generalized Hidden Markov Model for the recognition of Human Genes in DNA, in: Proc. ISMB-96 (AAAI/MIT Press, St. Louis, MO, 1996).
[16] B. Lowerre and R. Reddy, The Harpy speech understanding system, in: W. A. Lea, ed., Trends in Speech Recognition (Prentice Hall, 1980).

[17] Matlab. The Mathworks Inc., Natick, Ma., hitp://www.mathworks.com/products/matlab/

[18] I. T. Nabney, NETLAB: Algorithms for Pat- tern Recognition (Springer, Heidelberg, 2002). http:/www.ncrg,aston.ac.uk/netlab/

[19] [19] J. R. Quinlan, C4.5: Programs for Machine Learning, (Morgan Kaufmann, 1993).

[20] L. R. Rabiner, A tutorial on hidden Markov models and selected applications in speech recognition, in: Proc. IEEE, 77(2) (1989) $257-$ 286.

[21] S. Salzberg, X. Chen, J. Henderson and K. Fasman, Finding genes in DNA using decision trees and dynamic programming, in: Proc. ISMB96 (AAAL/MIT Press, St. Louis, MO, 1996).

[22] R. E. Schapire, The strength of weak learnability, Machine Learning 5 (1990) 197-227.

[23] C. E. Shannon, Communication in the presence of noise, in: Proc. IRE 37 (1949) 10-21.

[24] A. J. C. Sharkey, N. E. Sharkey, and G. O. Chandroth, Neural nets and diversity, Neural Computing and Applications 4 (1996) 218-227.

[25] J. Shavlik, Combining symbolic and neural learning, Machine Leaming $14(2)(1994) 321-331$

[26] T. A. Thanaraj, A clean data set of EST-confirmed splice sites from Homo sapiens and standards for clean up procedures, Nucleic Acids Research, 27(13) (1999) 2627-2637.

[27] T. A. Thanaraj, Positional characterization of false positives from computational prediction of human splice sites, Nucleic Acids Research, 28(3) (2000) 744-754

[28] A. Tickle, F. Maire, G. Bologna, R. Andrews, and J. Diederich, Lessons from past, current issues, and future research directions in extracting knowledge embedded in artificial Neural Networks, in: S. Wermter and R. Sun, eds., Hybrid Neural Systems (Springer Verlag, Berlin, 2000).

[29] E. C. Uberbacher and R. J. Mural, GRAIL seeks out genes buried in DNA sequence, Science 254 (1991) 805.

[30] V. Vapnik, The nature of statistical leaming theory (Springer, New York, 1995).

[31] M. L. Vaughn, Derivation of the multilayer perceptron weight constraints for direct network interpretation and knowledge discovery, Neural Networks 12(9) (1999) 1259-1271.

[32] J. D. Watson, N. H. Hopkins, J. W. Roberts, J. Argetsinger A. Weiner Molecular Biology of the Gene, 4th Ed.; (Benjamin Cummings, Menlo Park, CA, 1987).

[33] D. H. Wolpert, Stacked generalization, Neural Networks 5 (1992) 241 259.

[34] Y. Xu and E. C. Uberbacher, Gene prediction by pattern recognition and homology search, in: Proc. Fourth International Conference on Intelligent Systems for Molecular Biology (AAAI Press, 1996) 241-251.

[35] $T$, Yada and $M$. Hirosawa, Gene recognition in cyanobacterium genomic sequence data using the Hidden Markov Model, in: Proc. ISMB-96 (AAAV/MIT Press, St. Loulis, MO, 1996).

[36] S. Yang, A. Browne and P. Picton, Multistage Neural Network ensembles, in: Proc. Third Intemational Workshop on Multiple Classifier Systems (Cagliari, Italy) Lecture Notes In Computer Science 2364 (Springer, Heidelberg, 2002) 91-97.

[37] X. Ying and E. C. Uberbacher, Gene prediction by pattern recognition and homology search, in: Proc. ISMB-96 (AAAI/MIT Press, St. Louis, MO, 1996)

[38] http://www.spss.com/spssbi/clementine/ 